\title{
Impact of Physical Fitness and Nutritional Diet for Healthy Life- A Cross- Sectional Questionnaire Survey
}

\author{
Pon Preeja. R ${ }^{1}$ Leslie Rani. S ${ }^{2}$ and Brundha M.P ${ }^{3}$ \\ ${ }^{1}$ Graduate Student, Department of General Pathology, Saveetha Dental College, \\ Saveetha Institute of Medical And Technical Sciences, Chennai, Tamilnadu India \\ ${ }^{2}$ Tutor, Department of General pathology Saveetha Dental College, Saveetha Institute \\ of Medical And Technical Sciences, Chennai-600077, Tamil Nadu, India \\ ${ }^{3}$ Associate professor, Department of General Pathology, Saveetha Dental College, Saveetha \\ Institute of Medical And Technical Sciences, Chennai, Tamil Nadu India
}

\section{ABSTRACT}

Physical fitness is the ability of the body to carry out work without getting tired. Fitness is often achieved through proper nutrition, exercise, rest and moderate vigorous physical activity. Nutrition is vital , as it gives energy to carry the heavy workouts. The growing epidemic of obesity is linked to recent decline in physical activity levels. Maintaining the normal weight involves a good physical activity, in combination with dietary changes.The aim of the study is to assess the impact of physical fitness and nutritional diet to lead a healthy life.A cross sectional questionnaire study was conducted to 100 people of General Population in Thiruvalluvar district of Tamil Nadu. The obtained data were analysed through SPSS software. The results were depicted in the form of graphs.The results say that $38 \%$ of the respondents follow both exercise and a healthy diet, $29 \%$ of the respondents practice only regular exercise and visit the gym often and 31\% of the respondents stated they follow only a nutritional diet to maintain a healthy life.Fitness awareness had a significant and positive effect on physical fitness and healthy habits. The questionnaire survey shows that the General population was aware of the importance of physical fitness and nutritional diet to lead a stress free healthy life.

KEY WORDS: EXERCISE,HEALTHY LIFE, NUTRITIONAL DIET, PHYSICAL FITNESS.

\section{INTRODUCTION}

Physical fitness is the ability of the body to carry out work without getting tired. It is commonly obtained through proper nutrition, moderate-vigorous physical exercising and sufficient rest (Tremblay et al., 2010). Health is a state of bodily, intellectual and social wellbeing in which disease and infirmity are absent. It is

\section{ARTICLE INFORMATION}

*Corresponding Author: brundha.sdc@saveetha.com Received 7th Aug 2020 Accepted after revision 28th Sep 2020 Print ISSN: 0974-6455 Online ISSN: 2321-4007 CODEN: BBRCBA

Thomson Reuters ISI Web of Science Clarivate Analytics USA and Crossref Indexed Journal

\section{Clarivate
Analytics}

NAAS Journal Score 2020 (4.31) SJIF: 2020 (7.728)

A Society of Science and Nature Publication,

Bhopal India 2020. All rights reserved.

Online Contents Available at: http//www.bbrc.in/

Doi: http://dx.doi.org/10.21786/bbrc/13.8/165 referred to as the ability to maintain homeostasis and recovery from insults (Definitions of Health | Primer on Public Health Population, no date). It is a measure of the body's capability to function effectively and efficiently. It is defined as the state of health and well-being of an individual. Fitness is often achieved through proper nutrition, exercise, rest and moderate to vigorous physical act (Medina-porqueres et al., 2015).. Exercise improves muscle strength inside the lungs, heart, and whole body. It lowers blood pressure, improves circulation and blood flow inside the muscles (Booth, Roberts and Laye, 2012).

It is a behavioral interference that has shown first-rate promise in allaying signs and symptoms of depression. The effects of practical degeneration are enormous and greatly lessen the general fitness of paraplegics, particularly in the musculoskeletal and cardiovascular systems,

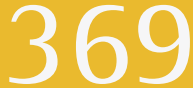


thereby increasing their hazard for cardiovascular disease(Caspersen, Powell and Christenson, 1985). In addition, exercise education has been stated to lower the resorptive process of the skeleton by decreasing bone and collagen catabolism and likely aiding in new bone formation (Martinsen, 1990). Physical activity is found to be a primary prevention against congestive heart failure, endothelial dysfunction, arterial dyslipidemia , hemostasis, deep vein thrombosis, cognitive dysfunction, despair and anxiety, osteoporosis, osteoarthritis, balance, bone fracture/falls, rheumatoid arthritis, colon cancer, breast cancer (Cowell, Squires and Raven, 1986).

Complete nutrition requires ingestion and absorption of vitamins, minerals, essential amino acids from protein and essential fatty acids from fat-containing food, also food energy in the form of carbohydrate, protein, and fat. Dietary habits and choices play a vital role in the quality of life, health and long life. Nutrition is the science that decodes the supplements and other material in food with respect to the maintenance, development,reprod uction, health and sickness.(Severson, 2010). Nutrition is vital and most effective for the development of human beings and animals and also for the prevention and remedy of disease. Nutrition is also essential to the preservation of appropriate fitness and functionality (Bell, 1988). In people, an undesirable food routine can create insufficiency related maladies, for example, frailty, scurvy, preterm birth, stillbirth and cretinism, or supplement overabundance, wellbeing compromising circumstances comprehensive of weight and metabolic disorder and some regular interminable fundamental sicknesses like cardiovascular infection, diabetes, and osteoporosis. Malnutrition can cause wasting of muscle in acute cases, and stunted growth are in chronic conditions (S. D. Ohlhorst et al., 2013).

Deficiencies can lead to quite a few fitness problems. These can encompass digestion problems, pores and skin disorders, stunted or defective bone growth, or even dementia(Whitney and Rolfes, 2005). Eating the right amount and exercising often can assist you avoid bloat and hold a healthy weight. Being physically lively is vital to achieve weight reduction goals. Even if you're not trying to lose weight, everyday exercise can improve cardiovascular health, boost your immune system, and increase your strength level (the Healthline Editorial Team, no date). A balanced diet is one that gives your body the supplements it needs to work accurately.To get the correct nourishment from your eating routine, you ought to expend most of your day by day calories as new organic products, vegetables, entire grains and vegetables, nuts, lean proteins('Healthy Lifestyle Habits Benefiting the Aging Body and Mind', 2011).

There are many literatures emphasizing the importance of physical inactivity as one of the most significant causes of reduced quality of life, disability, and death in the developed world. Some studies say maintaining a healthy body weight, or losing weight, is a direct function of calories consumed and expended (Patrick J. Skerrett, 2010). This study was conducted to assess the preference of the general population towards physical fitness and nutritional diet in order to maintain healthy lifestyle.

\section{MATERIAL AND METHODS}

The results were analysed by obtaining the percentages of each response using Microsoft excel and statistical analysis used was descriptive analysis using SPSS software.

Sample Selection: A cross- sectional questionnaire study was conducted among the general population of Sample size 100. The questions in subject were selected from various sources and compiled to required form. A preliminary set of questionnaires were given to the senior faculty members to validate the questions. Later implemented in the general population, to know how they understood the questions. These pre validated questions were implemented in the study.

Inclusion and Exclusion Criteria: Inclusion criterion set was general population, both males and females with age group moret than 18 years who are willing to participate in the study. Exclusion criterion:Chilren and people aged less than 18 years were excluded.

Sampling Method: In the present study,the sampling method used is a random sampling method.

Data Collection and Tabulation: The questionnaire which was taken on a survey planet and the responses were entered into the excel sheets and then tabulation of the data finally and the question comparison was done. The representation of the data is through the pie and bar graph .

Statistical Analysis: The statistical software used IBM SPSS V22.The statistical test used is Chi square test ( $p$ value).Type of analysis used were descriptive analysis,demographic data.

Figure 1: pie chart shows the result of the people participated in the study, 59\% were male (red) and 41\% were female respondents (blue)

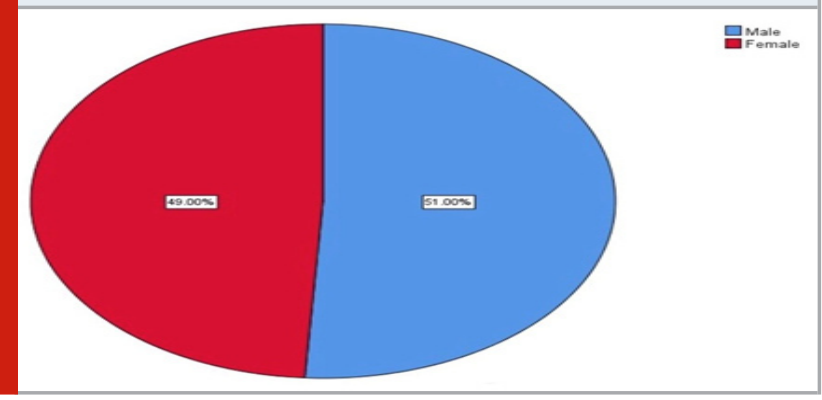

\section{RESULTS AND DISCUSSION}

Among 100 respondents, male participants are 51 and female participants are 49 (Figure 1). Based on the obtained results, the respondents are grouped in three sets. The first set of people stated they consume healthy 
food in order to stay healthy. Second set of people stated that they follow regular exercise and the third set of people stated that along with healthy food, practicing regular exercise improves the lifestyle and reduces the risk of cardiovascular diseases.

People who're physically energetic at a sufficient level attain a big range of bodily and mental health benefits in comparison to the ones who aren't lively (Blair et al., 1995) . Exercise may be a set of physical activity that's planned, structured, associated repetitive and has a final or an intermediate objective in the development or maintenance of body's health (Golden, 2002). Nutrition is also vital, not only for the growth and development of humans but also in the prevention and treatment of disease (Sarah D. Ohlhorst et al., 2013). Figure 2 shows that $14 \%$ of the respondents practice regular exercise and $15 \%$ of them go to gym, 31\% of the respondents eat only a healthy diet, $40 \%$ of the respondents follow both exercise and a healthy diet.

Figure 2: Pie chart shows various methods followed to maintain the fitness level.40\% of them follow exercise and follow a healthy diet(orange).14\% follow regular exercise(blue), whereas 15\% go to gym (red). 31\%eat only a healthy diet(green).

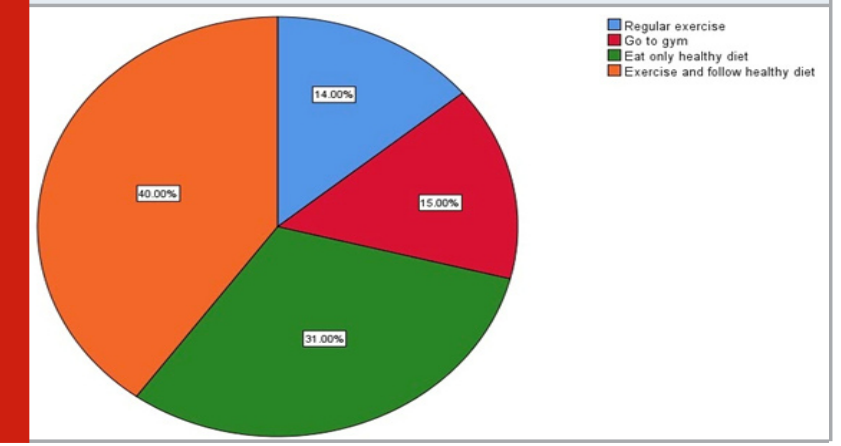

Figure 3:Bar graph represents the association between gender and the ways they maintain their fitness level.Xaxis represents gender and $\mathrm{Y}$ axis represents the count /number of responses.26\% of the females exercise and follow nutritional diet, which is an ideal way to attain healthy lifestyle ,whereas only $14 \%$ of the males follow healthy diet and exercise.Chi square test value is 12.721 , $p$ value is $0.005(p>0.05)$.Hence there is no statistical difference between the gender and various techniques in maintaining fitness level.

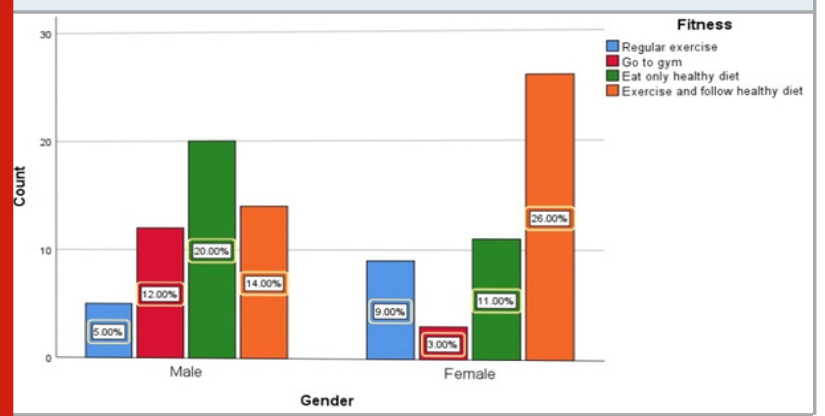

The people going to gym are advised to follow a healthy diet by gym instructors to maintain/improve the body build. Almost 2 million deaths per year were confirmed due to physical inactivity, thereby making physical activity the "best buy in public health"(Morris, 1994). $63 \%$ of them think that following a nutritional diet maintains the body health and $16 \%$ of them think that a nutritional diet alone can't maintain body health [figure 3]. However, many people know, more or less, were not aware of what healthy nutrition is and what is not. But this knowledge is not translated directly into behavior. They are not really following it in practice (Povey, 1999).

Figure 4: Pie chart shows 63\% of the respondents think that following a nutritional diet maintains body health(blue).16\% of the respondents do not agree with the fact that body health can be achieved by following a healthy $\operatorname{diet}(\mathrm{red}) .21 \%$ of the respondents have no idea(green).

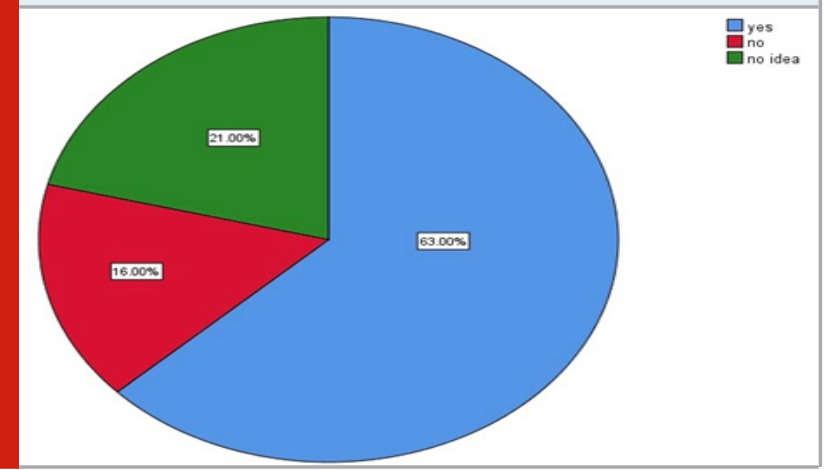

Figure 5: Pie chart shows that $41 \%$ of the respondents feel tired after consuming small amounts of food during exercise(blue).59\% of the respondents do not feel tired after consuming small amount of food (red).

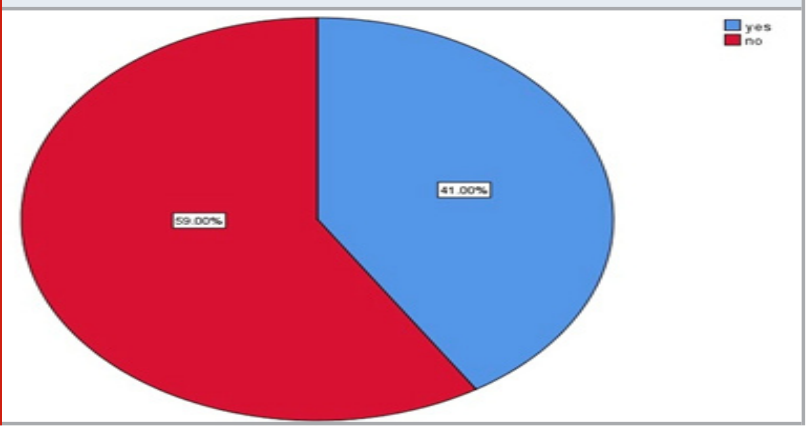

Figure 4 shows that $41 \%$ of the respondents feel tired after consuming a small amount of food during exercise. This might be due to inadequate intake of healthy drinks and nutritious supplements in between their practice sessions. 58\% of the respondents agree with the fact that one can lead a healthy life by only having a diet without exercise and 28\% of them don't agree with the fact having a nutritional diet without exercise can lead to a healthy life [figure 5]. The roles of nutrition and fitness, both singularly and together, helps in maintaining bodily functions, including cognitive, 
immune, skeletal, muscular, and other functions. When asked about the changes they made in their diet plan, $12 \%$ of the population have reduced the intake of sugar rich foods, 19\% of them have stopped consuming carbonated drinks [figure 6].

Figure 6: Pie chart shows that $58 \%$ of the respondents feel that one can lead a healthy life by following only a nutritional diet without exercising(blue).19\% of the respondents do not agree with this fact(red).23\% of the respondents have no idea(green).

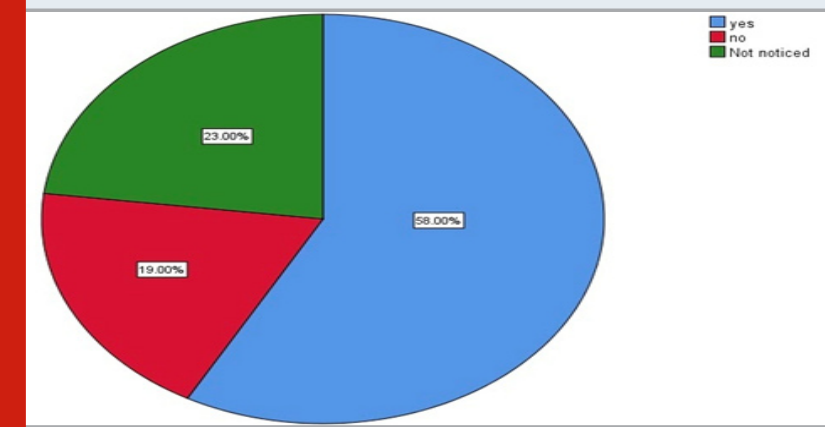

Figure 7: Pie chart shows the changes made by the respondents to achieve a healthy lifestyle .12\% of the respondents have reduced taking sugar foods(blue).19\% of the respondents have stopped having carbonated drinks(red).28\% of the respondents have stopped consuming protein rich foods(green). $41 \%$ of the respondents had their own ways of maintaining physical fitness(orange).

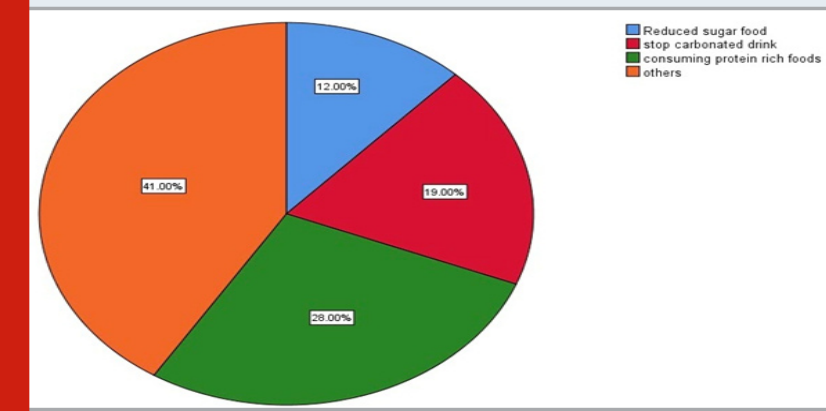

As because our diet has a direct impact on our muscle's ability to recover after a workout, we need to maintain a proper diet. 57\% of the participants think that motivated individuals have a healthier lifestyle [figure7]. Though the participants in the survey were aware of the benefits of doing regular physical activity and following a nutritional diet, few don't find time to practice regular exercise. They say following a balanced diet and consuming cholesterol free food makes them healthier and they don't often fall sick. This survey gets a mixed review on the practice to lead a healthy life.

\section{CONCLUSION}

Within the limitations of the questionnaire survey, we conclude that the majority of the population are aware of the importance of physical fitness and nutritional
Figure 8: The bar graph represents the association between gender and the changes the respondents made in their diet. The $\mathrm{X}$-axis represents the gender and $\mathrm{Y}$-axis represents the count/number of responses. $14 \%$ of the males stopped consuming protein carbonated drinks, whereas only 5\% of the females have reduced consuming carbonated drink.Red represents respondents who stopped drinking carbonated drinks.Chi Square test was done to find the variables.Chi square test value is $10.969 \mathrm{p}$ value is .012 ( $p>0.05)$. Hence there is no statistical significant difference between gender and changes made in the dietary planner.

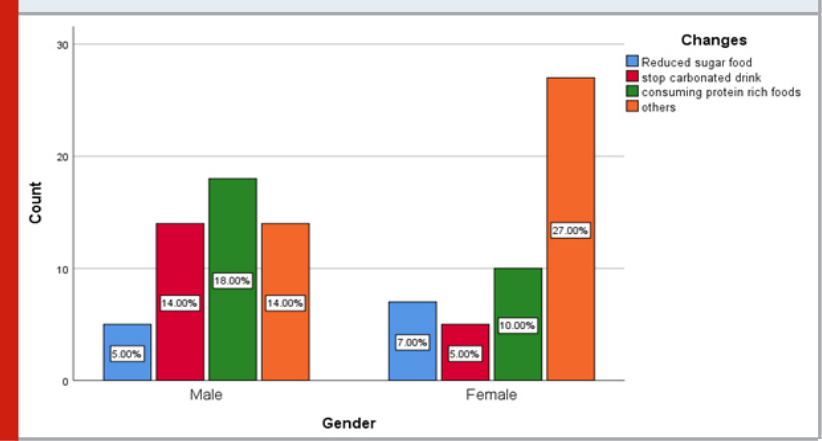

Figure 9: Pie chart shows that 57\% of the respondents think that the motivated individuals lead a healthier life (blue) $.27 \%$ of the respondents do not agree with this fact(28\%). $16 \%$ of the respondents have no idea (red).

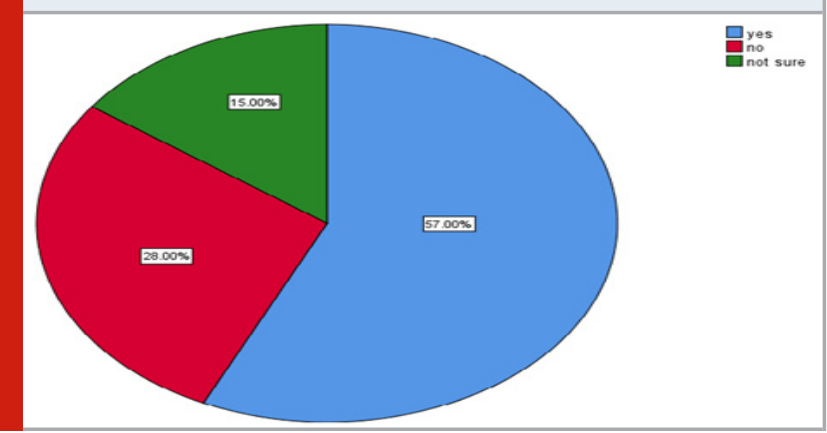

diet to lead a stress free healthy life. The females are more aware of fitness in relation to diet than the males. Fitness awareness had a significant and positive effect on physical fitness and healthy habits.

\section{ACKNOWLEDGEMENTS}

We thank Saveetha dental college for the support to carry out this survey.

Conflict of Interests: None to declare.

\section{REFERENCES}

Bell, S. J. (1988) ‘Book Reviews: Understanding Normal And Clinical Nutrition, 2nd Ed. E. N. Whitney, and C. B. Cataldo, with S. R. Rolfes. West Publishing Co.- College Et School Division, St. Paul, MN, 1987, 304 pages, 32.76; (Study Guide: 10.72 for nonfaculty; no charge 
for faculty)', Journal of Parenteral and Enteral Nutrition, pp. 107-107. doi: 10.1177/014860718801200122.

Blair, S. N. et al. (1995) 'Changes in physical fitness and all-cause mortality. A prospective study of healthy and unhealthy men', JAMA: the journal of the American Medical Association, 273(14), pp. 1093-1098.

Booth, F. W., Roberts, C. K. and Laye, M. J. (2012) 'Lack of exercise is a major cause of chronic diseases', Comprehensive Physiology, 2(2), pp. 1143-1211.

Caspersen, C. J., Powell, K. E. and Christenson, G. M. (1985) 'Physical activity, exercise, and physical fitness: definitions and distinctions for health-related research', Public health reports, 100(2), pp. 126-131.

Cowell, L. L., Squires, W. G. and Raven, P. B. (1986) 'Benefits of aerobic exercise for the paraplegic: a brief review', Medicine and science in sports and exercise, 18(5), pp. 501-508.

Definitions of Health | Primer on Public Health Population (no date). Available at: https://web. archive.org/web/20160812145405/http://phprimer. afmc.ca/Part 1-TheoryThinkingAboutHealth/ ConceptsOfHealthAndIllness/DefinitionsofHealth (Accessed: 30 June 2020).

Golden, M. H. N. (2002) 'The Development of Concepts of Malnutrition', The Journal of Nutrition, p. 2117S-2122S. doi: 10.1093/jn/132.7.2117s.

the Healthline Editorial Team (no date) Nutritional Deficiencies (Malnutrition): Symptoms and Treatment, Healthline. Healthline Media. Available at: https://www. healthline.com/health/malnutrition (Accessed: 30 June 2020).

Healthy Lifestyle Habits Benefiting the Aging Body and Mind' (2011) The Aging Intellect, pp. 63-88. doi: 10.4324/9780203882504-4.

Martinsen, E. W. (1990) 'Benefits of exercise for the treatment of depression', Sports medicine, 9(6), pp. 380-389.

Medina-porqueres, I. et al. (2015) 'Prospective, Multicenter Study Protocol to Adaptate and Validate the Simple Shoulder Test to Spanish Population', Journal of novel physiotherapies. OMICS International, 5(5), pp. $1-5$.

Morris, J. N. (1994) 'Exercise in the prevention of coronary heart disease', Medicine \& Science in Sports \& Exercise, p. 807???814. doi: 10.1249/00005768199407000-00001.

Ohlhorst, S. D. et al. (2013) 'Nutrition research to affect food and a healthy life span', The Journal of nutrition, 143(8), pp. 1349-1354.

Ohlhorst, S. D. et al. (2013) 'Nutrition research to affect food and a healthy lifespan', Advances in Nutrition, pp. 579-584. doi: 10.3945/an.113.004176.

Patrick J. Skerrett, W. C. W. (2010) 'Essentials of Healthy Eating: A Guide', Journal of midwifery \& women's health. NIH Public Access, 55(6), p. 492.

Povey, R. (1999) 'A critical examination of the application of the Transtheoretical Model's stages of change to dietary behaviours', Health Education Research, pp. 641-651. doi: 10.1093/her/14.5.641.

Severson, K. (2010) Told to Eat Its Vegetables, America Orders Fries. Available at: https://www.nytimes. com/2010/09/25/health/policy/25vegetables.html (Accessed: 30 June 2020).

Tremblay, M. S. et al. (2010) 'Physiological and Health Implications of a Sedentary Lifestyle', Applied physiology, nutrition, and metabolism = Physiologie appliquee, nutrition et metabolisme. Appl Physiol Nutr Metab, 35(6). doi: 10.1139/H10-079.

Whitney, E. N. and Rolfes, S. R. (2005) Understanding Nutrition. Wadsworth Publishing Company. 